\title{
Development of hypertension after live kidney donation
}

\author{
${ }^{1}$ Department of Internal Medicine-Nephrology, Keimyung University School of Medicine, Daegu, Korea
${ }^{2}$ Department of Internal Medicine-Nephrology, Ewha Womans University Seoul Hospital, Seoul, Korea
${ }^{3}$ Department of Biostatistics, Korea University College of Medicine, Seoul, Korea
${ }^{4}$ Department of Internal Medicine-Nephrology, Seoul National University Hospital, Seoul, Korea
}

Yaerim Kim ${ }^{1}$, Eunjung Kang ${ }^{2}$, Jina Park ${ }^{3}$, Sehoon Park ${ }^{4}$, Yong Chul Kim ${ }^{4}$, Yon Su Kim, Hajeong Lee ${ }^{4}$

Background: Hypertension is a common comorbidity and also a risk factor for end-stage kidney disease in living kidney donors. Herein, we aimed to evaluate the impact of exposure to overweight after donation on the development of new-onset hypertension.

Methods: Subjects took national health check-up $\geq 2$ times during 2001-2018 were included. After matching with multiple variables, controls were randomly extracted. Exposure to overweight and obesity was defined by body mass index $\geq 23 \mathrm{~kg} / \mathrm{m}^{2}$ and $\geq 25 \mathrm{~kg} / \mathrm{m}^{2}$ during follow-up period. Overweight/obesity status was divided into four groups; persistently no exposure, exposure at only last health check-up, persistently exposure in two times of health check-up, and recovered from exposure at last health check-up. We used a multivariate logistic regression model to identify risk factors for new-onset hypertension.

Results: A total of 1,642 donors and 3,655 controls were finally included in the study. During 7.6 \pm 3.1 years, there were 142 (8.6\%) and 253 (6.9\%) subjects newly diagnosed with hypertension, respectively. Kidney donation significantly increased risk for new-onset hypertension (adjusted odds ratio [aOR], 1.53; 95\% confidence interval [CI], 1.21-1.93). Persistent overweight significantly increased risk for new-onset hypertension (aOR, 3.53; 95\% Cl, 2.07-6.35 vs. aOR, 1.69; 95\% Cl, 1.19-2.43), whereas recovered from overweight did not increase risk (aOR, $1.61 ; 95 \% \mathrm{Cl}, 0.36-5.1 \mathrm{vs}$. aOR, $0.87 ; 95 \% \mathrm{Cl}, 0.35-1.87)$ in kidney donor and controls, respectively. Exposure to persistent obesity significantly increased the risk for hypertension in both groups, but recovered from obesity still increased the risk in kidney donors (aOR, 2.51; $95 \% \mathrm{Cl}, 1.03-5.45)$ in contrary to the control $(\mathrm{aOR}, 1.60 ; 95 \% \mathrm{Cl}$, 0.88-2.76).

Conclusions: Both exposures to overweight or obesity increased the risk for new-onset hypertension, but recovered from overweight or obesity showed different results in donors. Physicians need to be focused on counseling for reducing the modifiable risk factor such as for overweight after donation.

Corresponding author: Hajeong Lee

E-mail:mdhjlee@gmail.com

\section{(c) The Korean Society for Transplantation}

This is an Open Access article distributed under the terms of the Creative Commons Attribution Non-Commercial License (http://creativecommons.org/licenses/by-nc/4.0/) which permits unrestricted non-commercial use, distribution, and reproduction in any medium, provided the original work is properly cited. 